\title{
The Effects of Body Position on Chemotherapy-Induced Nausea and Vomiting: A Single-Blind Randomized Controlled Trial
}

\author{
Mohammad Fathi ${ }^{1}$; Alireza Nikbakht Nasrabadi ${ }^{1}$; Sina Valiee ${ }^{2, *}$ \\ ${ }^{1}$ School of Nursing and Midwifery, Tehran University of Medical Sciences, Tehran, IR Iran \\ ${ }^{2}$ School of Nursing and Midwifery, Kurdistan University of Medical Sciences, Sanandaj, IR Iran \\ ${ }^{*}$ Corresponding Author: Sina Valiee, School of Nursing and Midwifery, Kurdistan University of Medical Sciences, Sanandaj, IR Iran. Tel: +98-9188734619, Fax: +98-8716660092, E-mail: \\ valiee@muk.ac.ir
}

Received: January 22, 2014; Revised: March 7, 2014; Accepted: April 8, 2014

\begin{abstract}
Background:Chemotherapy is the cornerstone of cancer treatment; however, alongside therapeutic effects, nausea and vomiting are two common complications of chemotherapy.

Objectives: The aim of this study was to investigate the effects of body position on chemotherapy-induced nausea and vomiting.

Materials and Methods: This was a single-blind randomized controlled clinical trial. We recruited a convenience sample of 79 patients and randomly allocated them to either experimental or control groups. Patients in the control group received chemotherapy in supine position while the experimental group received chemotherapy in semi-Fowler's position. All patients were assessed for the severity, duration, and frequency of nausea and vomiting episodes every three hours up to 24 hours, ie, in nine time-points. Study data was analyzed bySPSS v. 16.

Results: The severity, duration, and frequency of nausea and the severity and frequency of vomiting episodes in the control group differed significantly across the nine measurement time-points $(\mathrm{P}<0.001)$. In the experimental group, the severity $(\mathrm{P}=0.254)$ and frequency of nausea $(\mathrm{P}=0.002)$ episodes as well as the frequency of vomiting $(\mathrm{P}=0.008)$ episodes differed significantly across the measurement timepoints. Moreover, the study groups differed significantly across the measurement time-point in terms of the severity $(\mathrm{P}<0.001)$, duration $(\mathrm{P}<0.001)$, and frequency of nausea $(\mathrm{P}=0.002)$ and the severity $(\mathrm{P}<0.001)$ and frequency $(\mathrm{P}<0.001)$ of vomiting episodes.

Conclusions: Compared to supine position, semi-Fowler's position is more effective in relieving chemotherapy-induced nausea and vomiting.
\end{abstract}

Keywords:Nursing; Cancer; Nausea; Vomiting

\section{Background}

Cancer is a major health problem worldwide (1). It caused more than 7.6 million death in 2008, a mortality rate of about $13 \%$. Most of these deaths happened in low-income and middle-income countries. It is estimated that by 2030 , cancer-related deaths increase to 13 million worldwide (2). Moreover, according to the American Cancer Society (2013), the number of new cases of cancer in the United States in 2013 will be 1.6 million (3). In Iran, cancer is the third leading cause of death after cardiovascular diseases and accidents (4). Chemotherapy is the cornerstone of cancer treatment (5). As a systemic therapy, it destroys cancer cells even at remote parts of the body $(6,7)$. Chemotherapy, which is currently given to more than $50 \%$ of patients with cancer, saves millions of lives and brings many patients back to life (8). In the United States, about one million patients with cancer undergo chemotherapy yearly (9). Alongside therapeutic effects, chemotherapy exerts many side effects (10, 11). According to Roffe and Ernst, 80\% of chemotherapies have some side effects. Due to its toxic effects, e.g. diarrhea, nausea, and vomiting, some patients consider chemotherapy unacceptable and unbearable (11). Sharma et al. reported that nausea and vomiting are respectively the first and the fourth most common side effects of chemotherapy (12). Firouzkuhi et al. also found that $54 \%$ to $96 \%$ of the patients receiving chemotherapy experienced nausea and vomiting $(13,14)$; however, $59 \%$ of adolescents participating in Baker and Ellett's study reported that the side effects resulted more from cancer itself rather than from cancer treatments (15).

Side effects of chemotherapy progressively worsen patients' condition and cause anxiety and depression and hence, can decrease patients' compliance with treatment regimens. Uncontrolled nausea and vomiting delay the administration of chemotherapy and significantly reduce patients' quality of life $(16,17)$. Hamadani et al. also found that $70 \%$ to $80 \%$ of the patients with cancer considered nausea

Implication for health policy/practice/research/medical education:

Compared to supine position, semi-Fowler's position is more effective in relieving chemotherapy-induced nausea and vomiting. Therefore, given the simplicity, safety, and cost-effectiveness of semi-Fowler position, healthcare providers can relieve chemotherapy-induced nausea and vomiting by placing patients in this position.

Copyright (c) 2014, Iranian Red Crescent Medical Journal; Published by Kowsar Corp. This is an open-access article distributed under the terms of the Creative Commons Attribution License, which permits unrestricted use, distribution, and reproduction in any medium, provided the original work is properly cited. 
Fathi M et al.

and vomiting as the most debilitating side effects of chemotherapy. Besides, $46 \%$ to $50 \%$ of the patients who had participated in their study were thinking about the withdrawal of treatment (18). Many prevention and treatment strategies have been developed to manage chemotherapy-induced nausea and vomiting. For instance, antiemetic medications such as serotonin 5-HT3-receptor antagonists can significantly decrease the incidence of nausea and vomiting (19). Nonetheless, the incidence of chemotherapy-induced nausea and vomiting is still as high as $60 \%$ to $72 \%(20)$. Moreover, antiemetic agents have, in turn, many debilitating side effects such as headache, constipation, fatigue, mouth dryness, dizziness, diarrhea, drowsiness, akathisia, and extrapyramidal signs and symptoms (19), which can aggravate patients' condition. Robertson et al. noted that there was no standard prophylactic treatment for preventing and managing chemotherapy-related complications (21). Consequently, nonpharmacological complementary and alternative therapies are currently administered, either alternatively or in combination with conventional treatments, for managing these complications (20). Nonpharmacological therapies prescribed for the management of chemotherapy-induced nausea and vomiting included, but not limited to, acupuncture, acupressure, relaxation, biofeedback, selfhypnosis, distraction, guided imagery, music therapy, and herbal medications such as Ginger $(22,23)$. An important nonpharmacological intervention for the management of chemotherapy-induced nausea and vomiting is patient positioning. Shahdadi etal. found that in comparison with supine position, semi-Fowler's position significantly reduced the frequency, severity, and length of nausea in patients undergoing hemodialysis (24). Robertson et al. also found that compared with supine position, post-myelography nausea and vomiting was less frequent and less severe in semi-Fowler's position (21). Rezaei-Adaryani et al. reported that positioning significantly decreased low back pain, improved physical comfort, and shortened the length of hospital stay after coronary angiography (25). Firouzkuhi et al. also reported that in comparison with supine position, patients receiving chemotherapy experienced fewer, shorter, and milder episodes of nausea and vomiting in semi-Fowler's position $(13,14)$. Despite these findings, there is no integrated standard protocol for patient positioning during chemotherapy in our country, Iran, and patients received chemotherapy mainly in supine position.

\section{Objectives}

This study aimed to investigate the effects of body positioning on chemotherapy-induced nausea and vomiting.

\section{Materials and Methods}

This was a single-blind randomized controlled trial conducted in 2011. The study setting was the referral oncology ward of a 350-bed general teaching hospital affiliated with Kurdistan University of Medical Sciences, Sanandaj, Iran. The study population consisted of all patients with cancer referred to the study setting for receiving chemotherapy. The inclusion criteria were being older than 18 years of age, a definite diagnosis of any types of cancer, receiving single-day chemotherapy courses, and the history of receiving at least two courses of chemotherapy. The exclusion criteria were inability to assume or remain in the supine and semi-Fowler's positions as well as need for emergency interventions during chemotherapy. Moreover, patients with electrolyte imbalance and pregnant women were excluded. Based on sample size formula, with delta of 1.4 , an alpha of 0.05 , and a power of 0.80 , the sample size was determined as 31 patients in each group. Accordingly, we recruited a convenience sample of 79 patients for the study. Patients were randomly allocated to either experimental or control groups by using the permuted block randomization design. All participants were blind to the group assignment. Patients in the control group underwent no position change. Accordingly, they received chemotherapy in supine position. In the experimental group, patients received chemotherapy while they were placed in semi-Fowler's position for three to four hours. All the patients in both groups received chemotherapy at a same time of day (from 8:00 to 14:00) and at a same environment. Moreover, the chemotherapy protocol and the nausea and vomiting treatment protocol were the same for both groups (Figure 1).

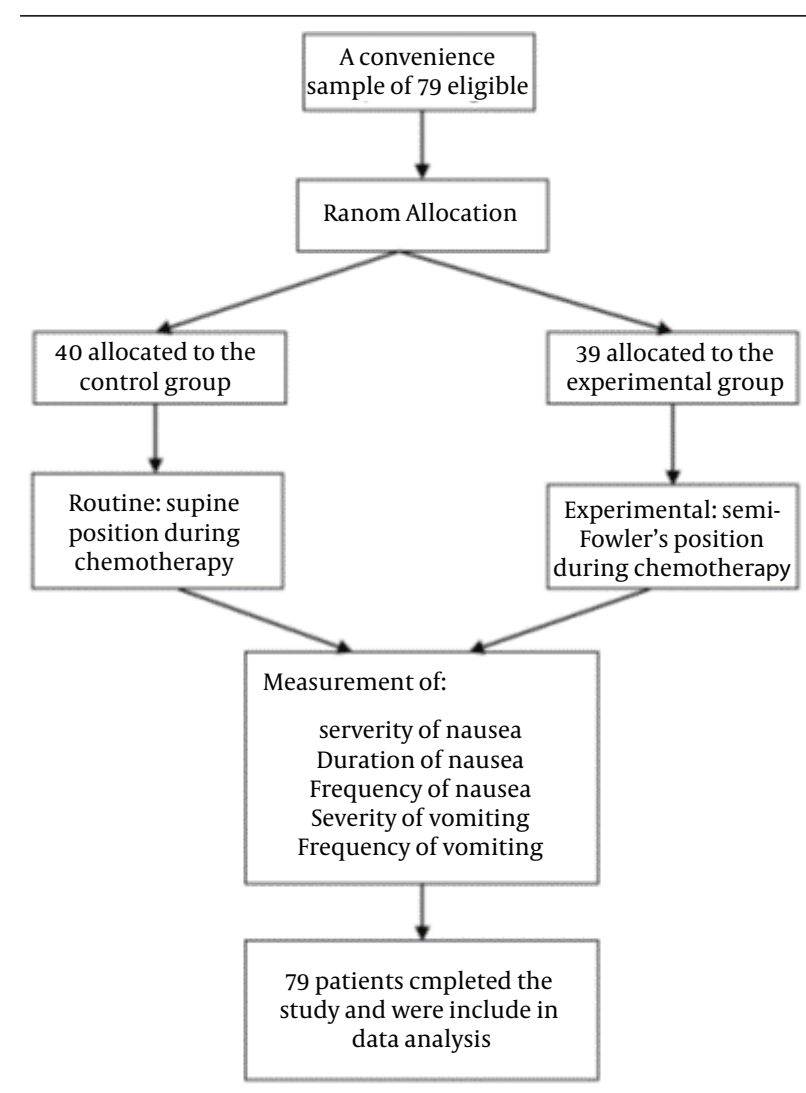

Figure 1. CONSORT Flowchart, Displaying the Flow of Subjects From Enrollment Through Analysis 
Fathi $M$ et al.

All patients were assessed for the severity, duration, and frequency of nausea episodes and the severity and frequency of vomiting episodes for 24 hours. The assessment time-points were immediately before chemotherapy and every three hours throughout and after chemotherapy. The study instrument consisted of a demographic questionnaire and a visual analogue scale (VAS) for assessing the severity of nausea and vomiting. The demographic and clinical data questionnaire was developed based on a literature review. Then, we invited ten nursing lecturers to assess the content validity of the questionnaire. The questionnaire was revised based on their comments. A standard VAS was used for evaluating the severity of nausea and vomiting episodes; in our study, VAS was a tencentimeter ruler on which zero stood for no nausea or vomiting and ten stood for the most severe nausea and vomiting. VAS is routinely used worldwide for assessing the severity of nausea and vomiting (26-30). Wood et al. also noted VAS as a valid and reliable tool for assessing subjective measures (26). We also assessed the frequency of nausea and vomiting episodes by observing and interviewing patients. The duration of nausea and vomiting episodes was measured by using a chronometer. Study data was analyzed by using the SPSS v.16.0 (SPSS Inc., Chicago, IL, USA). We assessed the normality of the study variables by using the Kolmogorov-Smirnov test. The result of this test revealed that all the study variables had a normal distribution. Accordingly, we employed parametric statistical tests for data analysis. We compared the study groups in terms of demographic variables by using the independent-samples $t$ and the Chi square tests. Finally, we employed the repeated-measures analysis of variance (repeated-measure ANOVA) test to compare the severity, duration, and frequency of nausea and vomiting both within groups and between groups across the nine measurement time-points. All statistical assumptions related to the repeated measure ANOVA test were fulfilled. The level of significance was set at below 0.05. The Institutional Review Board and Ethics Committee of Kurdistan University of Medical Sciences approved the study. Moreover, the study was registered at the Iranian Registry of Clinical Trials (http://www.irct.ir/) with the registration number of IRCT201111268208N1. A written informed consent was obtained from all the study participants.

\section{Results}

Totally, 79 patients participated in this study: 40 in the experimental and 39 in the control groups. The mean patients' age in the experimental and the control groups were $53.33 \pm 15.61$ and $56.67 \pm 13.44$, respectively. Most of the study participants (55.69\%) were females. The most common type of cancer among the study participants was stage IV cancer of alimentary system. Finally, the most common therapeutic regimen administered to our participants consisted of mitotic inhibitor agents. The results of the independent-samples $t$ and the Chi square tests revealed that the study groups did not differ significantly in terms of the demographic characteristics including age, gender, employment and educational status, and residency as well as clinical data including type and stage of cancer, length of disease, and treatment regimen (Tables 1 and 2)

\begin{tabular}{|c|c|c|c|}
\hline & Control Group & Experimental Group & PValue \\
\hline Age, $y^{a}$ & $53.33 \pm 15.61$ & $56.67 \pm 13.44$ & $0.31^{\mathrm{C}}$ \\
\hline Gender $^{\mathrm{b}}$ & & & $0.43^{C}$ \\
\hline Male & $16(40)$ & $19(48.7)$ & - \\
\hline Female & $24(60)$ & $20(51.3)$ & - \\
\hline Educational Status $^{\text {b }}$ & & & $0.09^{d}$ \\
\hline Illiterate & $22(55)$ & $27(69.2)$ & - \\
\hline Elementary & $11(27.5)$ & $3(7.7)$ & - \\
\hline Diploma & $3(7.5)$ & $6(15.4)$ & - \\
\hline Higher & $4(10)$ & $3(7.7)$ & - \\
\hline Employment $^{\mathrm{b}}$ & & & $0.57^{\mathrm{C}}$ \\
\hline Employed & $23(57.5)$ & $20(51.3)$ & - \\
\hline Unemployed & $17(47.2)$ & $19(48.7)$ & - \\
\hline Residency $^{\mathrm{b}}$ & & & $0.75^{c}$ \\
\hline City & $27(67.5)$ & $25(64.1)$ & - \\
\hline Rural & $13(32.5)$ & $14(35.9)$ & - \\
\hline $\begin{array}{l}\text { a Data are presented as me } \\
{ }^{c} \text { Chi square test. } \\
\text { b Date are presented as No } \\
\text { d Fisher's exact test. }\end{array}$ & & & \\
\hline
\end{tabular}


Fathi M et al.

\begin{tabular}{|c|c|c|c|}
\hline & Control Group & Experimental Group & P Value \\
\hline Type of Cancer & & & $0.67^{\mathrm{b}}$ \\
\hline Respiratory & $1(0.02)$ & $3(0.08)$ & - \\
\hline Gastrointestinal & $19(52)$ & $15(42)$ & - \\
\hline Breast and Gynecology & $3(0.08)$ & $2(0.05)$ & - \\
\hline Kidney & $3(0.08)$ & $6(0.17)$ & - \\
\hline Skin & $3(0.08)$ & $4(0.11)$ & - \\
\hline Others & $7(0.19)$ & $5(0.14)$ & - \\
\hline Stage of Cancer & & & $0.55^{\mathrm{C}}$ \\
\hline Primary & $12(44)$ & $11(36)$ & - \\
\hline Metastatic & $15(56)$ & $19(64)$ & - \\
\hline Period of Chemotherapy & & & $0.46^{\mathrm{C}}$ \\
\hline$<5$ & $20(55)$ & $26(68)$ & - \\
\hline $6-9$ & $12(33)$ & $8(21)$ & - \\
\hline$>10$ & $4(11)$ & $4(11)$ & - \\
\hline Interval of Chemotherapy & & & $0.82^{\mathrm{C}}$ \\
\hline 1-3 week & $14(37)$ & $12(34)$ & - \\
\hline $3<$ & $24(63)$ & $23(66)$ & - \\
\hline Treatment Regimen & & & $0.82^{\mathrm{C}}$ \\
\hline Alkylating Agents & $3(0.07)$ & $5(0.13)$ & - \\
\hline Antimetabolites & $8(0.2)$ & $9(0.23)$ & - \\
\hline Mitotic Inhibitors & $21(0.53)$ & $19(0.49)$ & - \\
\hline Topoisomerase Inhibitors & $8(0.2)$ & $6(0.15)$ & - \\
\hline
\end{tabular}

\begin{tabular}{|c|c|c|c|c|c|c|c|c|c|c|}
\hline & \multicolumn{2}{|c|}{ Severity of Nausea } & \multicolumn{2}{|c|}{ Duration of Nausea } & \multicolumn{2}{|c|}{ Frequency of Nausea } & \multicolumn{2}{|c|}{ Severity of Vomiting } & \multicolumn{2}{|c|}{$\begin{array}{l}\text { Frequency of } \\
\text { Vomiting }\end{array}$} \\
\hline & Con. & Exp. & Con. & Exp. & Con. & Exp. & Con. & Exp. & Con. & Exp. \\
\hline $\begin{array}{l}\text { Chemotherapy } \\
\text { Start }\end{array}$ & $0.27 \pm 0.81$ & $0.18 \pm 0.72$ & $2 \pm 3.55$ & $1.23 \pm 1.89$ & $0.25 \pm 0.63$ & $0.69 \pm 0.92$ & $0.08 \pm 0.35$ & $0.1 \pm 0.3$ & $0.2 \pm 0.68$ & $0.26 \pm 0.49$ \\
\hline 3 & $0.42 \pm 0.98$ & $0.46 \pm 0.91$ & $1.78 \pm 3.36$ & $0.97 \pm 2.1$ & $0.27 \pm 0.71$ & $0.31 \pm 0.69$ & $0.42 \pm 0.98$ & $0.44 \pm 0.85$ & $0.22 \pm 0.42$ & $0.18 \pm 0.45$ \\
\hline 6 & $1.3 \pm 1.92$ & $0.77 \pm 1.08$ & $1.48 \pm 3.87$ & $0.59 \pm 1.44$ & $0.45 \pm 0.87$ & $0.15 \pm 0.36$ & $1.08 \pm 1.55$ & $0.33 \pm 1.06$ & $0.6 \pm 1.21$ & $0.15 \pm 0.81$ \\
\hline 9 & $1.35 \pm 1.99$ & $0.33 \pm 1.03$ & $1.8 \pm 3.96$ & $0.31 \pm 1.62$ & $0.65 \pm 0.94$ & $0.08 \pm 0.35$ & $0.53 \pm 1.17$ & 0 & $0.25 \pm 0.58$ & 0 \\
\hline 12 & $0.6 \pm 1.42$ & $0.8 \pm 0.35$ & $0.83 \pm 2.41$ & $0.05 \pm 0.32$ & $0.25 \pm 0.58$ & $0.03 \pm 0.16$ & $0.53 \pm 1.46$ & 0 & $0.1 \pm 0.37$ & 0 \\
\hline 15 & $0.52 \pm 1.84$ & 0 & $0.4 \pm 1.66$ & 0 & $0.1 \pm 0.37$ & 0 & $0.13 \pm 0.79$ & 0 & $0.03 \pm 0.15$ & 0 \\
\hline 18 & $0.13 \pm 0.51$ & $0.15 \pm 0.96$ & $0.2 \pm 0.64$ & $0.41 \pm 2.56$ & $0.1 \pm 0.37$ & 0 & $0.2 \pm 0.72$ & 0 & $0.03 \pm 0.26$ & 0 \\
\hline 21 & $0.8 \pm 0.47$ & 0 & $0.8 \pm 0.35$ & 0 & 0 & 0 & $0.1 \pm 0.63$ & 0 & $0.8 \pm 0.26$ & 0 \\
\hline 24 & $0.15 \pm 0.58$ & $0.1 \pm 0.44$ & $0.13 \pm 0.56$ & $0.23 \pm 1.06$ & $0.03 \pm 0.15$ & 0 & 0 & 0.08 & 0 & 0 \\
\hline $\begin{array}{l}\text { P Value within- } \\
\text { subjects }\end{array}$ & 0 & 0.254 & 0 & 0.62 & 0 & 0.002 & 0 & 0.003 & 0 & 0.008 \\
\hline $\begin{array}{l}\text { PValue between- } \\
\text { subjects }\end{array}$ & 0 & & 0 & & 0.002 & & 0 & & 0 & \\
\hline
\end{tabular}

\footnotetext{
a Abbreviations: Con., control group; and Exp., experiment group.
} 
Fathi $M$ et al.

On the other hand, the results of the repeated-measures ANOVA test for the within-subjects factor of time revealed that the severity, duration, and frequency of nausea and the severity and frequency of vomiting episodes in the control group differed significantly across the nine measurement time-points $(\mathrm{P}<0.001$; Table 3$)$. The results of this test showed that in the experimental group, the severity $(P=0.003)$ and frequency $(P=0.002)$ of nausea episodes as well as the frequency of vomiting episodes ( $\mathrm{P}$ $=0.008$ ) differed significantly across the nine measurement time-points (Table 3). Moreover, the results of the repeated-measures ANOVA test for the between-subjects factor of group demonstrated that the study groups differed significantly across the nine measurement timepoint in terms of the severity, duration, and frequency of nausea and the severity and frequency of vomiting episodes (Table 3). The trend of changes also revealed that in comparison with supine position, semi-Fowler's position was more effective in reducing the severity, duration, and frequency of nausea and the severity and frequency of vomiting episodes (Table 3 ).

\section{Discussion}

The aim of this study was to investigate the effects of body positioning on chemotherapy-induced nausea and vomiting. Study findings revealed that in comparison with supine position, patients experienced less severe nausea in semi-Fowler's position. Firouzkuhi et al. and Shahdadi et al. also found that in contrast to supine position, nausea episodes were less severe in semi-Fowler's position $(13,14,24)$. These findings confirm the effectiveness of semi-Fowler's position in reducing the severity of chemotherapy-induced nausea. Moreover, the study findings demonstrated that in contrary to the patients in the control group, patients in the experimental group experienced shorter episodes of nausea. In addition, Firouzkuhi et al., Shahdadi et al. and Robertson et al. reported the same finding $(13,14,21,24)$. On the other hand, our study results showed that except for the T3 time-point, the trend of nausea frequency in the experimental group was downward. We also found that in comparison with the control group, nausea episodes were significantly less frequent in the experimental group. Shahdadi et al. also found that in contrast to the supine position, the number of hemodialysis-induced nausea episodes was significantly lower in semi-Fowler's position (24). Firouzkuhi et al. also reported the same finding for chemotherapy-induced nausea (13). These findings support the effectiveness of semi-Fowler's position in decreasing the frequency of chemotherapy-induced nausea.

Our study findings also revealed that the severity of vomiting episodes in the experimental group was significantly lower than the control group across all the nine measurement time-points. Firouzkuhi et al. also found that in comparison with supine position, vomiting episodes were less severe in semi-Fowler's position
(12). Moreover, our study findings demonstrated that the frequency of the vomiting episodes in the experimental group was significantly lower than the control group. Besides, from $\mathrm{T} 4$ to $\mathrm{T} 8$, patients in the experimental group experienced no episodes of vomiting. Firouzkuhi et al. also reported the same finding $(11,12)$.

To summarize, in comparison with supine position, patients in semi-Fowler's position generally experienced fewer, shorter, and milder episodes of nausea and vomiting. This can be attributed to many factors. In semi-Fowler's position, gravity lifts abdominal organs and structures away from diaphragm and stomach. Consequently, intra-gastric pressure and diaphragmatic movements decrease, which in turn relieve nausea and vomiting. Study findings indicated that in contrast to the supine position, semi-Fowler's position is more effective in reducing the severity, duration, and frequency of nausea and the severity and frequency of vomiting episodes in patients receiving chemotherapy. Given the simplicity, safety, and cost-effectiveness of semi-Fowler's position, healthcare providers can relieve chemotherapy-induced nausea and vomiting by placing patients in this position.

\subsection{Limitations and Recommendations}

We selected a convenience sample for this study. Accordingly, the study findings might have limited generalizability. Moreover, in this study, we compared the effectiveness of only two positions in relieving chemotherapy-induced nausea and vomiting. Consequently, investigating the effects of other body positions on chemotherapy-induced nausea and vomiting is recommended. Investigating the effects of body positioning on nausea and vomiting induced by other clinical conditions is also recommended.

\section{Acknowledgements}

The present study was the outcome of research proposal approved by Research Council of Kurdistan University of Medical Sciences. This research project would not have been possible without the collaboration of the patients; the authors appreciate all of them.

\section{Authors' Contributions}

The conception and design of the study, acquisition of data, analysis and interpretation of data: Mohammad Fathi, Alireza Nikbakht Nasrabadi, and Sina Valiee. Drafting the article or revising it critically for important intellectual content: Mohammad Fathi, Alireza Nikbakht Nasrabadi, and Sina Valiee. Final approval of the version to be submitted: Mohammad Fathi, Alireza Nikbakht Nasrabadi, and Sina Valiee.

\section{Funding/Support}

This study was supported by the research fund of the Kurdistan University of Medical Sciences. 


\section{References}

1. Baykal U, Seren S, Sokmen S. A description of oncology nurses' working conditions in Turkey. Eur J Oncol Nurs. 2009;13(5):368-75.

2. World Health Organization. Media centre Cancer: WHO; 2013. [updated 2014]. Available from: http://www.who.int/mediacentre/ factsheets/fs297/en/index.html.

3. American Cancer Society. [updated 2014]; Anonymos Cancer Facts \& Figures American Cancer Society. 2013. Available from: http://www.cancer.org/Research/CancerFactsStatistics/ CancerFactsFigures2013/2013-cancer-facts-and-figures.pdf.

4. Somi MH, Mousavi SM, Rezaeifar P, Naghashi SH. Cancer incidence among the elderly population in the Northwest of Iran: A population based study. Iran J Cancer Prev. 2012;2(3):117-26.

5. Karimi MH, Dehghan MP, Milani MJ. The influence of group consult on sexual health of patient with breast cancer. SJFM. 2006;11(40):201-6.

6. National Institutes of Health. Support for People With Cancer Chemotherapy and You: National Cancer Institute U.S. Department of Health and Human Services; 2007. [updated 2011]. Available from: www.cancer.gov/cancertopics/chemotherapy-and-you.pdf.

7. American Cancer Society. General types of treatment. 2008. Available from: http://www.Cancer.org/docroot/CRI-2-4-4X-local-VSSytemic-Therapy-5asp?sitearea=Accessed.

8. Ignoffo RJ, Rosenbaum EH. What happens in chemotherapy? .Everyone's Guide to Cancer Therapy: How Cancer Is Diagnosed, Treated and Managed Day to Day. 5th ed Kansas City: Andrews McMeel Publishing; 2002.

9. Advisory Committee. Center for Drug Evaluation and Research Gastrointestinal Drugs. 2003. Available from: www.fda.gov/ OHRMS.

10. Hawkins R, Grunberg S. Chemotherapy-induced nausea and vomiting: challenges and opportunities for improved patient outcomes. Clin J Oncol Nurs. 2009;13(1):54-64.

11. Roffe L, Schmidt K, Ernst E. A systematic review of guided imagery as an adjuvant cancer therapy. Psychooncology. 2005;14(8):607-17.

12. Sharma R, Tobin P, Clarke SJ. Management of chemotherapyinduced nausea, vomiting, oral mucositis, and diarrhoea. Lancet Oncol. 2005;6(2):93-102.

13. Firouzkuhi MR, Mazlom SR, Firozi ET, Kikhaei A. Effects of semifowler position on nausea and vomiting during chemotherapy for cancer patients. Tabibeshargh Journal. 1999;1(2):25-9.

14. Firouzkuhi MR, Mazloum SR. The Effect of Semi-Upright Position on Incidence and Intensity of Chemotherapy-Induced Nausea and Vomiting. IJMS. 2003;28(4):206.

15. Baker PD, Ellett ML. Measuring nausea and vomiting in adolescents: a feasibility study. Gastroenterol Nurs. 2007;30(1):18-28.

16. Campos de Carvalho E, Martins FT, dos Santos CB. A pilot study of a relaxation technique for management of nausea and vomiting in patients receiving cancer chemotherapy. Cancer Nurs.
2007;30(2):163-7.

17. Yoo HJ, Ahn SH, Kim SB, Kim WK, Han OS. Efficacy of progressive muscle relaxation training and guided imagery in reducing chemotherapy side effects in patients with breast cancer and in improving their quality of life. Support Care Cancer. 2005;13(10):826-33.

18. Hamadani M, Chaudhary L, Awan FT, Khan JK, Kojouri K, Ozer H, et al. Management of platinum-based chemotherapy-induced acute nausea and vomiting: is there a superior serotonin receptor antagonist? J Oncol Pharm Pract. 2007;13(2):69-75.

19. Klein J, Griffiths P. Acupressure for nausea and vomiting in cancer patients receiving chemotherapy. Br J Community Nurs. 2004;9(9):383-8.

20. Baggott CR. Nursing care of children and adolescents with cancer: WB Saunders Co; 2002.

21. Robertson WD, Lapointe JS, Nugent RA, Robinson RG, Daly LF Positioning of patients after metrizamide lumbar myelography. AJR Am J Roentgenol. 1980;134(5):947-8.

22. American Cancer Society.[updated 2014]; Nausea and vomiting. 2011. Available from: http://www.cancer.org/treatment/treatmentsandsideeffects/physicalsideeffects/dealingwithsymptomsathome/caring-for-the-patient-with-cancer-at-home-nauseaand-vomiting.

23. King CR. Nonpharmacologic management of chemotherapyinduced nausea and vomiting. Oncol Nurs Forum. 1997;24(7 Suppl):41-8.

24. Shahdadi H, Mazloum SR, Hidari A, Badakhsh M. Investigation effect of semi-folwer on hemodialysis complications. Sci J Nurs Midwifery Mashhad. 2009;9(1):31-6.

25. Rezaei-Adaryani M, Ahmadi F, Asghari-Jafarabadi M. The effect of changing position and early ambulation after cardiac catheterization on patients' outcomes: a single-blind randomized controlled trial. Int J Nurs Stud. 2009;46(8):1047-53.

26. Wood JM, Chapman K, Eilers J. Tools for assessing nausea, vomiting, and retching. Cancer Nurs. 2011;34(1):E14-24.

27. Meek R, Kelly AM, Hu XF. Use of the visual analog scale to rate and monitor severity of nausea in the emergency department. Acad Emerg Med. 2009;16(12):1304-10.

28. Braude D, Soliz T, Crandall C, Hendey G, Andrews J, Weichenthal L. Antiemetics in the ED: a randomized controlled trial comparing 3 common agents. Am J Emerg Med. 2006;24(2):177-82.

29. Grimsehl K, Whiteside JB, Mackenzie N. Comparison of cyclizine and ondansetron for the prevention of postoperative nausea and vomiting in laparoscopic day-case gynaecological surgery. Anaesthesia. 2002;57(1):61-5.

30. Moore JK, Elliott RA, Payne K, Moore EW, St Leger AS, Harper NJ, et al. The effect of anaesthetic agents on induction, recovery and patient preferences in adult day case surgery: a 7-day follow-up randomized controlled trial. Eur J Anaesthesiol. 2008;25(11):876-83. 